Books, videos, cd-roms, dvds and any other relevant items submitted for a review in the BDJ should be addressed to:

Mike Grace, Editor, British Dental Journal, 64 Wimpole Street WIG 8YS

\section{Human Physiology: The Basis of Medicine (2nd edition)}

\author{
G. Pocock, C.D. Richards and M. de Burgh Daly \\ Oxford: Oxford University Press, 2004 \\ price $€ 35$, pp714 \\ ISBN 0198585276
}

Why would dentists want to buy a physiology book, and why should they choose this one?

This book is aimed at first and second year medical students (who report that it is useful), although it is suitable for dental and science students. The demand among qualified dentists will be more limited, although it will be useful for those preparing for exams such as the MFD/S.

The content -31 chapters - is similar to other physiology books. The chapters progress from cellular physiology, through nerve, muscle and the major body systems, to sections on integrated functions (including regulation of growth, blood glucose, acid-base balance and the physiology of exercise). The final chapter on clinical physiology covers ageing and the pathophysiology of heart failure, hypertension, renal failure and liver failure.

On the whole, the content is good, although some topics (eg cardiovascular, respiratory and renal systems) are covered in more detail than others. The dental reader will be gratified by the good coverage of salivation, and also by the inclusion of information on trigeminal nerve pathways. The detailed section on neuromuscular transmission does, surprisingly, fail to mention 'botox'. But in spite of this, the content is generally applicable to contemporary practice.

The text is illustrated by numerous diagrams, some of them describing 'classical' physiology. Most of the diagrams are excellent, but some are too small, and because of this the detail is indistinct. This problem is especially evident in diagrams of nerve connections in reflex pathways. There are good pictures illustrating clinical conditions, such as hormonal imbalances, although the subjects should have been anonymised.

Text boxes provide useful summaries at the end of each section (students particularly like these features). Some self-test questions (and the answers!) are provided at the end of each chapter. However, other books do this better, often by means of a CD-ROM or accompanying workbook. I found very few typographical and no factual errors. The index is sufficient, but not comprehensive. Although the text uses North American spelling, the book redeems itself by using SI units.

Is it worth buying? Yes, it is. It provides a comprehensive coverage of human physiology at a level that is clinically applicable to the European audience. But if you want zappy goodies, such as CD-ROMs, then you should look elsewhere.

R. Orchardson

\section{Odontogenic Tumours and Allied Lesions}

P.A. Reichart, H.P. Philipsen
Surrey: Quintessence, 2004
price $£ 106$, pp388
ISBN 1850970599

This is an excellent text providing a very detailed and up-to-date review of odontogenic tumours and allied lesions. Written by highly respected experts in the relevant fields, it covers odontogenic tumours as classified in July 2003, and also provides relevant information on a variety of other related disorders such as fibrous dysplasia, cherubism and aneurysmal bone cysts.

The opening chapters are concerned with the classification of odontogenic tumours, normal odontogenesis and the radiological investigation of such lesions. The book then comprises chapters that consider each of the relevant lesions in some detail. In particular, each chapter provides discussion of terminology, clinical and radiological features, epidemiology, aetiological, pathogenesis, treatment and long-term behaviour of each lesion.

The information is well presented, good radiological images are provided, and the histopathological images generally delineate relevant features. When relevant, examples are given of the intra-oral manifestations of a particular disorder.

The chapters dealing with allied lesions are also well presented. I found the sections considering cherubism, fibrous dysplasia and melanotic neuroectodermal tumour of infancy particularly interesting and informing.

The authors have certainly achieved the goal of presenting a well written, accurate and helpful dissertation of odontogenic tumours. The sections considering treatment and long-term behaviour should be highly relevant to oral and maxillofacial surgeons, whereas sections concerned with histopathological features are clearly of interest to both general and oral and maxillofacial pathologists

Within the introduction the authors suggest that the readership may include undergraduates. I think this is highly unlikely as the text is far too detailed for undergraduate curricula. Certainly, however, most interested postgraduate students and specialists involved in the diagnosis, investigation and treatment of odontogenic tumours should find this book essential reference material.

This is an excellent text; Professors Reichart and Philipsen have done a splendid job.

S.R. Porter 

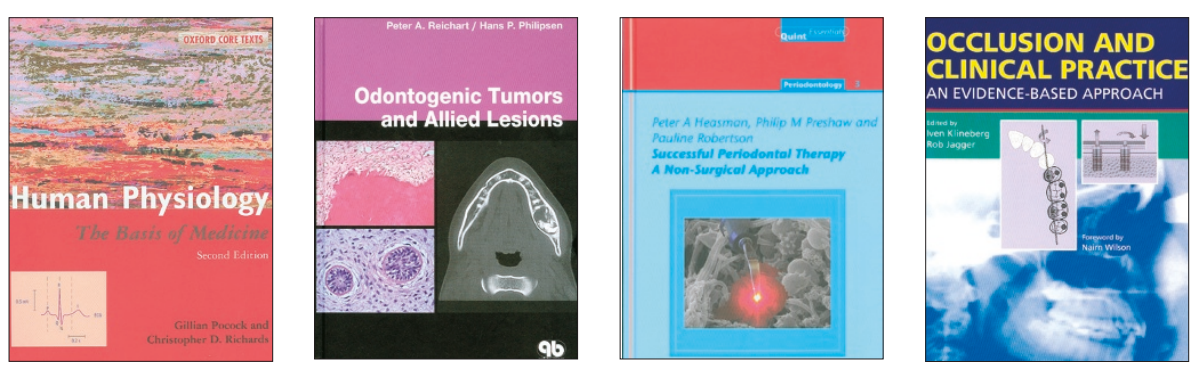

\section{Successful Periodontal Therapy: A Non-Surgical Approach}

\author{
P.A. Heasman, P.M. Preshaw, and P. Robertson \\ Surrey: Quintessence, 2004 \\ price $€ 28$, pp 118 \\ ISBN 1850970742
}

There is no shortage of publications seeking to guide the dentist in the provision of periodontal care. As a new and valuable contribution to dental education, the Quintessentials of Dental Practice series, of which this guide to non-surgical periodontal therapy is the latest volume, specifically aims at the busy general dental practitioner and his or her team. The authors are to be congratulated on the content, which goes a long way to improving routine periodontal therapy in practice. This very easy to read book forms a handy reference to be kept in the practice, whilst the layout, photographs and diagrams all help to constitute a wellproportioned text.

Clearly, in a book of this size, it cannot be the aim to provide a comprehensive review of treatment. As a result the authors are perhaps over selective in the choice of scientific papers that they discuss and that are suggested as further reading. However, they have generally fulfilled their stated aims, although there is little discussion of the possible limitations of non-surgical therapy, which is promised in the preface.

Overall, the book seems to make use of a sound evidence base, although the chapter on managing local risk factors, which includes a fairly extensive treatise on the role of occlusal factors, might be questioned in this respect. Similarly, there are some unfortunate inconsistencies in the text. For example, it is stated that 'the post-treatment morphology of the gingival tissue is often irregular and may further complicate plaque control' whilst, two pages later, the caption to the accompanying photograph states that 'the gingival shrinkage that has occurred will make it easier for the patient to use interdental aids to maintain a high level of plaque control, even though the gingival morphology is irregular'. The chapter on Hygiene Phase therapy is very welcome but concentrates a little too much on tools with little emphasis on the problems of patient motivation which is perhaps one of the hardest things most dentists and hygienists have to do each day.

One of the limitations of such a small volume is the need to refer to other volumes in the series, without which the reader is left with a number of questions. With this in mind, the book is nonetheless worth buying in its own right and will be of particular help for general dental practitioners and their hygienists.

G.J. Smart

\section{Occlusion and Clinical Practice - An Evidence Based Approach}

\author{
I. Klineberg, R. Jagger \\ London: Elsevier, 2004 \\ price $€ 145$, pp32.99
}

ISBN 0723610924

This is a superb book. I thoroughly enjoyed it and like most readers, even those with a special interest in occlusion and TMD, will realise they have learned a considerable amount from the wealth of information contained therein.

It is laid out in a very 'user friendly' manner, each chapter having boxes at the start containing firstly, a synopsis of the chapter and secondly, bullet points denoting the key facts contained in each chapter.

The book is divided into three sections and 15 chapters in total. The first section is comprised of four chapters dealing with Biological Considerations of the Occlusion.
The basic science is dealt with here, covering general cranio-facial growth and development of the occlusion, jaw movements and anatomy and physiology of the temporomandibular joint in both health and disease. Occlusal inter-arch relationships are dealt with in a concise and clear yet authoritative manner and make the understanding of complex mandibular movements readily understood.

The second section of this book deals with Assessment of the Occlusion and takes the reader step by step through a comprehensive clinical occlusal assessment and delineates what records should be kept, and their relevance to clinical practice. There is a very clear, well illustrated chapter on articulators, face bow systems and other occlusal records.

The largest section of the book, which comprises half of the text, is devoted to clinical matters and is entitled Clinical Practice and the Occlusion. This is presented in a very logical manner and deals with each of the different disciplines in dentistry in turn. These range from temporomandibular disorders, both arthrogenous and myogenous, occlusion and periodontal health, orthodontics, fixed and removable prosthodontics and implant restoration. There are two final chapters which deal with occlusal splints and the role of occlusal adjustment.

This book is edited by Iven Klineberg and Rob Jagger and has contributions from 15 internationally recognised authors who, each in their own section, provide an excellent overview. This book is well illustrated throughout in black and white and, whilst an authoritative text, makes easy and enjoyable reading.

The views of the authors, whenever possible, appear to be evidence based and therefore, as all dentists in every discipline are involved in occlusion, this should become an invaluable addition to the book shelf both for reference and as a clinical guide and not just for those with a 'special interest'!

R.J.M. Gray 